Meta

Journal des tradlucteurs

Translators' Journal

\title{
Problématique des équivalences sémantiques et de la traduction dans des dictionnaires arabe-français
}

\section{Rachid Lyadri}

Volume 42, numéro 1, mars 1997

Lexicologie et terminologie

URI : https://id.erudit.org/iderudit/003526ar

DOI : https://doi.org/10.7202/003526ar

Aller au sommaire du numéro

Éditeur(s)

Les Presses de l'Université de Montréal

ISSN

0026-0452 (imprimé)

1492-1421 (numérique)

Découvrir la revue

Citer cet article

Lyadri, R. (1997). Problématique des équivalences sémantiques et de la traduction dans des dictionnaires arabe-français. Meta, 42(1), 142-146. https://doi.org/10.7202/003526ar
Résumé de l'article

Cet article traite de la problématique de la traduction français / arabe ainsi que des difficultés rencontrées par les usagers du dictionnaire bilingue arabe / français à la recherche d'un équivalent adéquat. On se base sur la comparaison des entrées arabes Walafa, ya>isa, nazala, kanaza et bâla dans quatre dictionnaires. On peut distinguer trois types d'équivalents qui reviennent avec une certaine régularité dans les quatre dictionnaires $\mathrm{A} / \mathrm{F}$. 


\title{
PROBLÉMATIQUE DES ÉQUIVALENCES SÉMANTIQUES ET DE LA TRADUCTION DANS DES DICTIONNAIRES ARABE-FRANÇAIS
}

RACHID LYADRI

Université Hassan II, Casablanca, Maroc

\begin{abstract}
Résumé
Cet article traite de la problématique de la traduction français / arabe ainsi que des difficultés rencontrées par les usagers du dictionnaire bilingue arabe / français à la recherche d'un équivalent adéquat. On se base sur la comparaison des entrées arabes Walafa, ya ${ }^{>}$isa, nazala, kanaza et bāla dans quatre dictionnaires. On peut distinguer trois types d'équivalents qui reviennent avec une certaine régularité dans les quatre dictionnaires $A / F$.
\end{abstract}

\section{Abstract}

This article addresses problems related to French-Arabic translation and the difficulties encountered by users of Arabic-French dictionaries in search of appropriate equivalents. A comparison of five Arabic entries in four dictionaries reveals three types of recurrent equivalents.

\section{LES ÉQUIVALENTS PÉRIPHRASTIQUES}

Seul le $S A B I L$ échappe en quelque sorte à cette règle en illustrant des équivalents en français plus ou moins courts (courts au sens de: composés de deux ou trois unités dans la plupart des cas).

1 Entrée A

$y a>i s a$

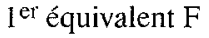

Désespérer

$2^{e}$ équivalent $F$, etc.

1 unité

1 unité

re espoir de

3 unités

En revanche, les trois autres dictionnaires ont une tendance lexicographique plutôt «explicative» de l'entrée arabe. On ne lui donne pas un équivalent français précis, mais on l'explicite :

1

Le Kazimirski
Entrée A walafa

$\begin{array}{ll}\text { Le } F . D . & \text { walafa } \\ & 1 \text { unité } \\ \text { Le D.A.F. } & \text { walafa } \\ & 1 \text { unité }\end{array}$

1 phrase $F$

Briller coup sur coup par des éclats de lumières répétés et qui se suivent sans interruption (se dit de l'éclair).

Soit 16 unités françaises (sans compter l'exemple pour l'unité arabe)

Briller coup sur coup

4 unités

Produire des jets de lumière

5 unités

Notons au passage que le F.D. (dont les équivalents sont souvent tirés du Kazimirski, comme nous l'avons constaté dans nos grilles d'observation) s'est rendu compte que l'équivalent fourni par le Kazimirski était effectivement trop long. Pour y remédier, il l'a réduit de 16 à 4 unités: «Briller coup sur coup». 
Les équivalents périphrastiques ne sont pas, contrairement à ce qu'on pourrait penser, opposés uniquement aux entrées «chargées» sémantiquement. Ils concernent tout aussi bien les verbes simples au niveau de la compréhension.

$\begin{array}{lll}\text { Le Kazimirski } & \text { nazala } & \text { Descendre d'un endroit plus élevé sur un endroit plus bas } \\ & 1 \text { unité } & 11 \text { unités }\end{array}$

Cela justifie donc la tendance lexicographique que nous évoquions plus haut. Au lieu de traduire nazala par une seule unité, descendre, le dictionnaire en donne 11 et qui n'éclairent pas davantage, à notre avis, la signification de l'entrée. Le lecteur se retrouve donc face à un certain nombre d'équivalents qui sont soit redondants, soit pléonastiques (notre dernier exemple).

Il est certain que ce type d'équivalents posent problème à au moins deux niveaux. D'abord, au niveau du code écrit : il est en effet quasiment impossible pour un étudiant en version, par exemple, de traduire une unité par une périphrase. L'équilibre de la version serait rompu s'il substituait l'une à l'autre. En outre, il obtiendrait un texte sémantiquement incohérent en français. C'est donc là un premier handicap pour l'étudiant. Ensuite, au niveau du discours, c'est-à-dire de la pratique de la chose acquise : cet étudiant saisit la signification de l'entrée arabe dans la langue française, mais a du mal à mettre en pratique cet acquis lexical, étant donné le caractère général de l'équivalent. En d'autres termes, le lexicographe, en fournissant une équivalence périphrastique, tend plus à éclairer le lecteur sur le mot de la langue source qu'à l'aider à en faire usage dans la langue cible.

Nous pensons qu'il serait souhaitable que les auteurs de dictionnaires bilingues A/F prennent conscience de ce problème d'insubstituabilité des unités. Darbelnet (1970: 92-95) manifeste le même avis quand il constate que les lexicographes: «ne mettent pas l'usager suffisamment en garde contre les méprises qui résultent de la non-superposition des aires sémantiques» dans leurs dictionnaires. Ils doivent également s'efforcer d'éviter ces longues phrases pour traduire un mot. Des équivalents courts et précis seraient certainement plus adaptés aux besoins de la traduction.

\section{LES ÉQUIVALENTS NON CONTEXTUELS}

L'observation des informations nous révèle l'irrégularité du traitement que les quatre dictionnaires réservent aux entrées qu'ils ont à traduire hors contexte.

Rappelons tout d'abord que cette irrégularité du traitement n'est pas propre aux dictionnaires $\mathrm{A} / \mathrm{F}$, mais qu'elle peut être décelée dans tout autre dictionnaire bilingue. Il s'agit donc là d'une problématique propre à la lexicographie différentielle. Nous verrons cela plus loin. Mais qu'en est-il des dictionnaires qui nous occupent? Prenons, par exemple, l'entrée nazala dans le D.A.F. On s'aperçoit qu'elle est traduite de la façon suivante :

nazala Descendre. Baisser. Faire halte. Prendre position.

1 entrée Gîter, Bivouaquer. Relâcher. Assiéger (avec <alà = sur). Maltraiter (avec bì = avec). Tomber à bas de (avec < an = de). Renoncer à. Céder, Concéder, Abdiquer $($ avec $<$ an $=$ de $)$.

En tout: 14 équivalents.

Il est évident que cette foule d'équivalents, ainsi livrée «en vrac», ne peut que déconcerter le lecteur: 14 équivalents sont proposés pour une seule entrée arabe. Ils sont utilisés les uns après les autres en n'étant séparés que par quelques points et virgules. Cela revient à dire qu'un traducteur qui dispose dans son support écrit d'un contexte précis se voit proposer 14 possibilités différentes de traductions. Laquelle choisir? Une solution cependant: les tester toutes afin de s'assurer de la bonne correspondance des contextes. 
Seulement, le traducteur dispose-t-il d'un temps suffisant pour effectuer toutes ces opérations? Et les équivalents correspondront-ils à son besoin? Gak (1970:115) note à ce propos que :

L'usager d'un dictionnaire bilingue est amené à constater souvent, et malheureusement trop souvent, que les équivalences fournies par le dictionnaire ne peuvent s'appliquer aux contextes réels.

Il s'agit donc bien là d'un véritable problème. Il est d'autant plus aigu que certains équivalents français n'ont, à première vue, aucun lien sémantique entre eux : Baisser, prendre position, bivouaquer peuvent effectivement paraître au lecteur comme trois entités différentes.

Il arrive cependant que l'équivalent soit illustré avec une occurrence d'emploi qui cerne davantage son champ de spécificité : c'est le cas du F.D.:

Kanaza: Enfouir, cacher sous terre (un trésor). Serrer qqch (dans un sac). Ficher en terre (une lance).

La présentation, entre parenthèses, des compléments d'information, peut effectivement orienter le lecteur dans son choix. Mais là encore, celui-ci ne risque-t-il pas d'être aussi embarrassé que dans l'exemple précédent, si l'équivalent sémantique qu'il cherche en français ne rentre pas dans les trois occurrences proposées par le lexicographe?

Enfouir/cacher sous terre un trésor

Serrer dans un sac

Ficher en terre une lance

Nous avons également constaté que la traduction hors contexte conduit parfois les lexicographes à deviner en quelque sorte les différents équivalents d'une entrée. Cela a pour conséquence d'entraîner une grande diversité au niveau des informations fournies. Cellesci peuvent en effet changer d'un dictionnaire à l'autre, et ce, pour la même entrée: pour Kanaza, nous avons recensé dans les quatre dictionnaires une dizaine d'équivalents potentiels:

Kanaza: Accumuler, amasser, empiler, entasser, tasser, cacher sous terre, enfouir sous terre, ficher en terre, serrer quelque chose, envelopper avec soin.

Ces différents équivalents mis ensemble parlent d'eux-mêmes. On remarquera seulement qu'un lecteur A traduira par accumuler ou entasser s'il consulte le $S A B I L$ et un lecteur B par envelopper avec soin ou serrer s'il consulte le D.A.F. Cela corrobore ce que nous soutenions, à savoir l'illustration d'équivalents différents selon les dictionnaires, due à une traduction hors contexte.

À ce sujet, nous souscrivons entièrement à l'avis d'Opkala (1979: 221) qui pense que :

La plupart des accusations contre le dictionnaire bilingue sont issues du fait que les équivalents qu'il fournit sont pour la plupart en mots isolés...

Cela dit, que peut-on faire face à cette situation lexicographique qui est pour le moins délicate et qui pose problème à tous les lexicographes bilingues?

Il est bien évident qu'on ne peut demander à ceux-ci de se substituer à l'usager et de deviner toutes les combinaisons possibles et imaginables qui peuvent se poser dans un dictionnaire bilingue. Dubois (1970:13) rappelle à juste titre que :

Le lexique est si vaste et les informations possibles si variées qu'il ne peut [le lexicographe], prétendre en un seul ouvrage satisfaire du même coup les besoins de n'importe qui. 
Cela est d'autant plus vrai que les mots qu'il a à traduire obéissent à un certain environnement et $y$ puisent en quelque sorte leur sens. Il est donc extrêmement difficile de les traduire en faisant abstraction de cet environnement qui confère la véritable acception au terme (voir Seleskovitch 1968: 143).

Pour régler ce problème, certains proposent d'expliciter le sens des vocableséquivalents, en montrant par exemple aussi bien leurs différences que leurs similitudes. Bref, une définition en quelque sorte des traductions (voir Darbelnet 1970: 29).

D'autres préconisent la multiplicité des contextes (Lyons 1970: 334). En d'autres termes, on illustrerait un équivalent avec les différents champs de spécificité qu'il est susceptible d'avoir dans la langue. Quant à Rey-Debove (1970:78), elle a son point de vue sur la question:

Nous pensons personnellement qu'un dictionnaire bilingue devrait présenter au moins un double système d'équivalents: pour un dictionnaire $A / F$, une définition de l'entrée $A$ en langue $\mathrm{F}$ et un équivalent lexical de $\mathrm{A}$ en langue $\mathrm{F}$. L'idéal serait un système quadruple [...], la confrontation de deux monolingues.

Ce sont donc là quelques suggestions formulées par les dictionnaires pour résoudre le problème des équivalences dans les dictionnaires bilingues. Elles méritent toutes d'être prises en considération par les lexicographes. Une seule réserve cependant: il ne faudrait pas que ces mesures entraînent un accroissement excessif des articles, car cela rendrait selon nous le dictionnaire difficile à consulter et les informations qui s'y trouvent longues à lire.

\section{LES ÉQUIVALENTS NON ISOMORPHIQUES}

Nous nous contenterons ici de citer un seul exemple très significatif, et qui donnera à notre avis une idée claire sur ce que peut être le non-isomorphisme entre les langues arabe et française dans certaines situations linguistiques. Cet exemple, nous l'avons tiré d'une entrée (bàla «uriner») qui nous servira pour l'étude des niveaux de langue. Il s'agit d'une expression arabe que le Kazimirski illustrait en tant que dérivé de l'entrée : Yastabilu l asad. Le $S A B I L$ la traduit ainsi :

yastabilu l>asad: prendre le taureau par les deux cornes, et le Kazimirski:

yastabilu l>asad: Il est capable d'aller se faire uriner dans la main par le lion (se dit d'un homme brave).

Nous pouvons constater ici que le $S A B I L$ a tenté, de trouver dans la langue française une expression plus ou moins équivalente de ce dérivé arabe, et y a réussi. Or, dans le Kazimirski, nous avons ce qu'on peut appeler un calque linguistique, à savoir une traduction mot à mot des unités arabes en français. Cela a pour conséquence de brouiller la saisie sémantique de cette expression arabe. En effet, compte tenu de la différence notionnelle des deux systèmes, un locuteur français ne serait pas en mesure de décoder la signification de: «Il est capable d'aller se faire uriner dans la main par le lion».

Pour pouvoir faire le lien sémantique, il faudrait que ce lecteur ait, entre autres, quelques notions de la culture arabe. Ladmiral (1972:28) met l'accent sur cet aspect «civilisationnel»:

... en dehors de ces facteurs socio-culturels qui interviennent au niveau «subjectif» de la langue maternelle, il faut souligner l'importance «objective» ou thématique de ce qu'on pourrait appeler la composante «civilisationnelle» qui intervient beaucoup plus au niveau de la «compétence-source» que de la langue maternelle. Si l'on est en mesure de traduire en anglais «the river» par le français «la Tamise» [...], ce n'est pas en fonction d'une compétence exclusivement linguistique mais en utilisant certaines connaissances de la civilisationsource qui font partie de ce que nous appelons la compétence «périlinguistique». 
Le SABIL a donc en quelque sorte utilisé dans ses articles cette compétence «périlinguistique» dont parle Ladmiral, en allant chercher dans la culture française l'expression «prendre le taureau par les cornes» comme équivalent. C'est en suivant cet exemple que les lexicographes bilingues seraient à même d'éviter le non-isomorphisme entre les langues arabe et française. Or, jusqu'à présent, les dictionnaires $\mathrm{A} / \mathrm{F}$ se sont plutôt bornés à réunir des équivalents sans trop se préoccuper de leur limite et surtout de leur cohérence dans la culture cible.

Ne serait-il pas préférable de tester d'abord l'efficacité de la traduction française avant de la lexicaliser définitivement dans l'article?

\section{RÉFÉRENCES}

DARBELNET, J. (1970) : «Dictionnaires bilingues et lexicographie différentielle», Langages, Paris, Larousse, $\mathrm{n}^{\circ} 19$, pp. $92-95$ et p. 29

DUBOIS, J. (1970) : «Dictionnaire et discours didactique», Langages, Paris, Larousse, n 19, p. 13.

GAK, V. G. (1970) : «La langue et le discours dans un dictionnaire bilingue», Langages, Paris, Larousse, $\mathrm{n}^{\circ} 19$, p. 115.

GALISSON, R. (1979) : «Lexicographie différentielle à l'institut de la mission de Thessalonique», ELA, 18

LADMIRAL, J.-R. (1972) : «La traduction et l'institution pédagogique», Langages, Paris, Larousse, $\mathrm{n}^{\circ} 28$, p. 28.

LYADRI, R. (1987) : Dictionnaire bilingue Arabe / Franç̧ais : problématique lexicographique, Thèse de doctorat, Strasbourg.

LYONS, J. (1970): Linguistique générale: introduction à la linguistique théorique, Paris, Larousse, p. 334.

OPKALA NWUGO, B. (1979): «Le dictionnaire bilingue et l'apprentissage de la langue seconde», Langages, Paris, Larousse, $\mathrm{n}^{\circ} 19$, p. 221.

REY-DEBOVE, J. (1970) : «Le domaine du dictionnaire», Langages, Paris, Larousse, $n^{\circ} 19$, p. 28.

SELESKOVITCH, D. (1968): L'interprète dans les conférences internationales, Paris, Lettres modernes, p. 143.

TAMBA, I. (1987): «Contraintes lexicales et pragmatiques du lexique : application aux problèmes de traduction japonais / français», Colloque de Strasbourg sur l'enseignement et l'apprentissage du lexique en français langue étrangère, 13-14 mars. 